\title{
Quantum dynamics of complex molecular systems
}

\author{
William H. Miller* \\ Department of Chemistry and K. S. Pitzer Center for Theoretical Chemistry, University of California, and Chemical Sciences Division, \\ Lawrence Berkeley National Laboratory, Berkeley, CA 94720-1460
}

Edited by Bruce J. Berne, Columbia University, New York, NY, and approved March 11, 2005 (received for review November 1, 2004)

This Perspective presents a broad overview of the present status of theoretical capabilities for describing quantum dynamics in molecular systems with many degrees of freedom, e.g., chemical reactions in solution, clusters, solids, or biomolecular environments.

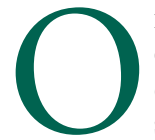

ne of the present challenges of molecular-level theory is to extend to complex molecular systems (i.e., those with many degrees of freedom) the great success it has had in the realm of "small molecule chemical physics." Thus, the methodology for carrying out state-to-state reactive scattering calculations to provide the complete description of chemical reactions of small molecular systems (about three or four atoms), and for performing wavepacket propagation (i.e., solving the time-dependent Schrödinger equation) to describe photodissociation or other laser-induced processes in such systems, is well in hand and widely applied these days. I do not mean to imply that such calculations are totally routine and uninteresting - the number of even small molecular systems that have been treated quantitatively is itself quite small, probably less than 10 , and new phenomena are still being discovered in such systems-so there is much interesting work yet to be done in this arena. But with all of the emphasis and interest in "nano" and "bio" phenomena these days, one would like to be able extend theoretical capabilities to be able to carry out reliable quantum dynamics simulations for chemical reactions, photoinduced dynamics, etc., in solution, in clusters, on surfaces, and in biomolecular environments.

\section{Is It Necessary?}

When talking with many colleagues on this topic I often get a "Why bother?" response, suggesting that classical molecular dynamics (MD) simulations are adequate, that quantum effects will surely average out in these complex systems and thus be unimportant. " ("Why are you worried about the third significant figure when we are still trying to get the first significant figure correct?") I am sure that this point of view is correct sometimes (maybe even most of the time), but one certainly knows that it is not always the case. For example, consider the water molecule, which is ubiquitous in bio simulations: the zero-point energy in its two $\mathrm{OH}$ stretching vibrations and its bending vibration is more than 20 times $k_{\mathrm{B}} T$ (at $300 \mathrm{~K}$ ). If this energy were allowed to behave classically in a simulation with hundreds (or thousands) of water molecules, it is clear that nonsense would result; the energy could leak out of these modes classically (which it cannot do quantum mechanically) and boil the system!

Of course, those that perform classical MD simulations are well aware of this, and water is usually treated as a rigid molecule that can rotate and thus realign its dipole moment and solvate a reaction coordinate appropriately, for example, but that is not allowed to vibrate. But often in an $\mathrm{H}$ atom transfer reaction-for example, in a biomolecular system, $\mathrm{AH}+\mathrm{B} \rightarrow \mathrm{A}+\mathrm{HB}$, where an $\mathrm{H}$ atom moves from site $\mathrm{A}$ to site $\mathrm{B}$ - it is not the $\mathrm{H}$ atom on $\mathrm{A}$ that winds up on B, but rather an intervening water molecule acts as a catalyst: $\mathrm{AH}+$ $\mathrm{OHH}+\mathrm{B} \rightarrow \mathrm{A}+\mathrm{HOH}+\mathrm{HB}$. To describe this process, one must obviously be able to make and break $\mathrm{OH}$ bonds, i.e., the $\mathrm{OH}$ bond degrees of freedom must be treated fully dynamically, and the quantum aspects of its dynamics will surely be significant.

So there are clearly situations in which the quantum mechanical aspects of the dynamics in complex systems will be important; the point of a simulation, after all, is for it to tell one the answer, not to have one guess the answer beforehand. If one performs only classical MD simulations, one will never know whether quantum effects are important. One must have the ability to include quantum effects into an MD simulation, even if only approximately, to know when they are important and when they are not. [None of this discussion, of course, is meant as a criticism of any of large amount of very impressive classical simulation work that has been carried out; one must walk before one can run. The goal is to build on the vast capabilities of classical MD to try to take the next step forward in theoretical methodology.]

\section{What Can Be Done?}

Speaking very broadly (as is being done in this Perspective), there are only two classes of computational methods that are feasible for general systems with many degrees of freedom: Monte Carlo path integral methods to evaluate the Boltzmann operator, $\exp (-\beta \hat{H})$, and thus describe equilibrium phenomena, and classical MD for describing realtime dynamical processes. ${ }^{\ddagger}$ I will discuss these in turn.

Path Integration for the Boltzmann Operator. The ability to evaluate the Boltzmann operator by Monte Carlo path integral methods (2) obviously makes it possible to evaluate essentially any kind of equilibrium property of a molecular system, and this methodology has advanced to the state that it can be applied to molecular systems with hundreds (perhaps thousands) of atoms. All of the tricks used in classical equilibrium calculations-e.g., various types of "free energy perturbation" methods $(3,4)$, Metropolis sampling schemes, and many other new sophisticated Monte Carlo sampling algorithms (5) — can readily by applied to fully quantum equilibrium calculations. Improvement to Monte Carlo methodology is continuing at a rapid pace, making it possible to apply these approaches to ever more complex molecular systems.

It is not apparent, however, that such calculations can be of any use for describing dynamical processes, but in some cases they in fact are of use. Most dynamical quantities of interest for complex systems can be expressed in terms

This paper was submitted directly (Track II) to the PNAS office.

*E-mail: millerwh@berkeley.edu.

${ }^{\dagger}$ Many investigators deal with systems so complex that any atomistic description is overly detailed, but this is too far afield for the present discussion.

${ }^{\ddagger}$ As soon as one makes such sweeping statements, exceptions immediately come to mind! In this regard I should mention the impressive, fully QM calculations that Makri and her students have carried out using a Feynman path integral formulation to integrate out a harmonic bath (for a review, see ref. 1). Though this approach is possible only for this very special type of system, the calculations this group has carried out are proving to be of immense value in providing benchmark results for model systems of many degrees of freedom.

() 2005 by The National Academy of Sciences of the USA 
of some kind of time correlation function $(6,7)$,

$$
C_{A B}(t)=\operatorname{tr}\left[\hat{A} e^{i \hat{H} t / \hbar} \hat{B} e^{-i \hat{H} t / \hbar}\right],
$$

where operator $\hat{A}$ typically involves the Boltzmann operator (and some other operator as well). [This is the quantum analog of the classical expression,

$$
\begin{array}{r}
C_{A B}{ }^{C \ell}(t)=(2 \pi \hbar)^{-f} \int d \mathbf{p}_{0} \int d \mathbf{q}_{0} \\
A\left(\mathbf{p}_{0}, \mathbf{q}_{0}\right) B\left(\mathbf{p}_{t}, \mathbf{q}_{t}\right),
\end{array}
$$

where $\left(\mathbf{p}_{0}, \mathbf{q}_{0}\right)$ are the initial momenta and coordinates for classical trajectories, and $\left(\mathbf{p}_{t}, \mathbf{q}_{t}\right)$ are the values at time $t$ that have evolved from these initial conditions. $A(\mathbf{p}, \mathbf{q})$ and $B(\mathbf{p}, \mathbf{q})$ are the classical functions that correspond to operators $\hat{A}$ and $\hat{B}$, and $f$ is the number of degrees of freedom.] For example, the flux-flux autocorrelation as given by Miller, Schwartz, and Tromp (8), the integral of which gives the rate constant for a chemical reaction, corresponds to operators $\hat{A}$ and $\hat{B}$ being

$$
\begin{aligned}
\hat{A} & =e^{-\beta \hat{H} / 2} \hat{F} e^{-\beta \hat{H} / 2} \\
\hat{B} & =\hat{F},
\end{aligned}
$$

where $\hat{F}$ is the flux operator for a "dividing surface" that separates reactants from products. A path integral evaluation of the time evolution operators, $\exp ( \pm i \hat{H} t / \hbar)$, however, is not straightforward because of the "sign problem," but such methods are possible for evaluating the flux correlation function at imaginary time $i \tau$ (where $\tau$ is real),

$$
\begin{aligned}
C_{f f}(i \tau)= & \operatorname{tr}\left[\hat{F} e^{-(\beta / 2+\tau / \hbar) \hat{H}}\right. \\
& \left.\cdot \hat{F} e^{-(\beta / 2-\tau / \hbar) \hat{H}}\right],
\end{aligned}
$$

because the time evolution operators become Boltzmann-like operators (which can be combined with the Boltzmann operators as indicated). The strategy is thus to compute $C_{f f}(i \tau)$ for real values of $\tau$ by Monte Carlo path integral methods and then use these values to obtain $C_{f f}(t)$ for real values of $t$ by numerical analytic continuation. A number of such approaches have been successfully implemented (9-11) [most recently in the work of Rabani et al. (11), using maximum entropy methods], and they typically give useful results for short times, which is sufficient to obtain the rate constant if the reaction mechanism is reasonably "direct," i.e., it does not involve any long time recrossing behavior (which is usually the case for reactions in complex systems, provided the "dividing surface" is well chosen).
Another theoretical approach recently developed for thermal rate constants of "direct" reactions is the quantum instanton (QI) model (12-14). At a low enough temperature for tunneling effects to be dominant, it involves two dividing surfaces (quantum analogs of the two turning point surfaces of the imaginary time trajectory in the semiclassical instanton theory) and provides an excellent description of reaction rates. It is also wholly expressed in terms of the Boltzmann operator (but involves no numerical analytic continuation) and can thus be readily evaluated by using Monte Carlo path integral methods. A recent calculation (14) using the QI approach has been carried out for a model of proton transfer in a polar solvent,

$$
A H+B \rightarrow A^{-}+H^{+} B
$$

in 255 methyl chloride molecules, thus demonstrating its applicability to complex systems.

Real-Time Dynamics. To describe longer time dynamical phenomena quantum mechanically requires that one deal explicitly with the time evolution operator, $\exp (-i \hat{H} t / \hbar)$, for real-time $t$. Because it is possible to evaluate it for systems with a few degrees of freedom (cf., "small molecule chemical physics"), one popular strategy for complex systems is a "mixed quantum-classical" approach, whereby a few of the most important degrees of freedom are treated quantum mechanically and the (many) less important degrees of freedom are treated classically, with the two being coupled in some approximate fashion. There is much excellent work going on in this area, both in very sophisticated applications to biomolecular systems and in new methodological developments. Some of the approaches are based on "localized quantum transitions," and others use a variety of wavepacket (usually Gaussian) methods. Some these approaches [e.g., the multiconfiguration time-dependent Hartree (MCTDH) and various Gaussian wavepacket expansion methods] are formulated within an "exact" framework, i.e., they would in principle lead to exact quantum results if the basis sets, etc., could be expanded without limit. The annual meeting of the American Chemical Society this past August in Philadelphia had a week-long symposium on quantum/classical calculations in chemistry and biophysics that contained many excellent papers in this area, and a recent workshop of the European Center for Atomic and Molecular Calculations was on a similar topic, Development of
Methods for Quantum Dynamics in the Condensed Phase. So there are a variety of things that can, and are, being done in this area ${ }^{\S}(15-38)$.

\section{Using Semiclassical Theory to Add Quantum Effects to Classical MD}

I will conclude this overview with the writer's prerogative of describing some of my own group's work in the area of quantum dynamics of complex systems, specifically in using semiclassical (SC) theory to add quantum effects to classical MD simulations. Numerically computed classical trajectories are the input to SC theory, so one is effectively trying to leverage the power of classical MD to add in the effects of quantum mechanics (within the semiclassical approximation). From what was learned from the large amount of semiclassical work in the 1970s (for a review of some of this early work, see refs. 39 and 40), it is clear (at least to me) that this will provide a very good description of quantum effects in the dynamics of complex molecular systems; the real question is whether one can make it practical enough to be applicable to systems with many degrees of freedom. Our goal, therefore, has been to formulate SC theory in such a way that its computational implementation is as close as possible to that of ordinary classical MD simulations.

The first step in this regard is to use the initial value representation (IVR) of semiclassical theory (41-44), whereby the nonlinear boundary value problem that normally arises in SC theory is replaced by an average (Monte Carlo) over the initial coordinates and momenta of classical trajectories (very much what one has to do in classical MD). I reviewed SC-IVR methodology and some of its applications only a few years ago (45), so there is no need to go over this in detail. Suffice it to say that the time evolution operator is given within the SC-IVR by a phase space average over the initial conditions of classical trajectories

$$
\begin{aligned}
e^{-i \hat{H} t / \hbar}= & (2 \pi \hbar)^{-F} \int d \mathbf{p}_{0} \int d \mathbf{q}_{0} C_{t}\left(\mathbf{p}_{0}, \mathbf{q}_{0}\right) \\
& \cdot e^{i S_{t}\left(\mathbf{p}_{0}, \mathbf{q}_{0}\right) / \hbar}\left|\mathbf{p}_{t}, \mathbf{q}_{t}\right\rangle\left\langle\mathbf{p}_{0}, \mathbf{q}_{0}\right|,
\end{aligned}
$$

where $\left(\mathbf{p}_{0}, \mathbf{q}_{0}\right)$ are the initial conditions for classical trajectories, $\left(\mathbf{p}_{t}, \mathbf{q}_{t}\right)$ are the momenta and coordinates at time $t$ that have evolved from these initial conditions, $S_{t}$ is the classical action integral along this trajectory, and $C_{t}$ is a preex-

\footnotetext{
$\S$ | will surely forget and omit some excellent and relevant work here-so my apologies before hand. This list is meant to be representative and is certainly not complete.
} 
ponential factor that involves derivatives of the values of $\mathbf{p}_{t}$ and $\mathbf{q}_{t}$ with respect to $\mathbf{p}_{0}$ and $\mathbf{q}_{0}$. The states $\left|\mathbf{p}_{0}, \mathbf{q}_{0}\right\rangle$ and $\left|\mathbf{p}_{t}, \mathbf{q}_{t}\right\rangle$ are coherent states (minimum uncertainty wavepackets) in the Herman-Kluk (46-48) version of the IVR as written above, or simply positions eigenstates $\left(\left|\mathbf{q}_{0}\right\rangle\right.$ and $\left.\left|\mathbf{q}_{t}\right\rangle\right)$ in the Van Vleck IVR.

For systems with many degrees of freedom, the integral over initial conditions in Eq. 5 is of high dimension ( $2 \mathrm{~F}$ for $\mathrm{F}$ degrees of freedom), so that Monte Carlo integration methods are mandatory if one is going to be able to apply it. Classical mechanics (e.g., classical time correlation functions corresponding to the quantum one in Eq. 1) also requires an average over the phase space of initial conditions, but this is more difficult for the SC-IVR because of the phase factor in the integrand of Eq. 5, which leads to oscillatory behavior (the semiclassical version of the "sign problem"). All recent efforts to make the SC-IVR approach practical have thus been focused on ways of dealing with this issue. I will mention just three "tricks" that have emerged recently and made a significant contribution to at least taming, if not completely solving, the SC sign problem.

The most generic trick goes under the name of "stationary phase Monte Carlo" (49) (the more descriptive moniker) or "modified Filinov filtering" (50) [from the researcher (51) in whose paper I first learned about it]. It employs a Gaussian filtering scheme to eliminate high-frequency oscillations from the integrand (which make little net contribution to the integral) and leads to a Monte Carlo procedure that favors the regions of "stationary phase" of the integrand. One can in fact view it as a numerical procedure that tunes continuously between the stationary phase approximation to the integral and the original (unfiltered) integral. It does not require that one find the stationary phase points explicitly, the (Metropolis) Monte Carlo procedure doing the job implicitly. The most evolved of such algorithms is the "generalized Filinov transformation" of Wang et al. (52).

A second trick $(53,54)$ that has proved extremely effective in some applications is to time average the integrand of Eq. 5. This process is an identity (i.e., entails no approximation) and has the effect of smoothing out the oscillations of the integrand, making the subsequent Monte Carlo average over initial conditions much more efficient, and the time averaging process requires little extra effort-one carries it out in the process of computing each trajectory-and is only a $1 d$ integral. In favor- able cases it has reduced the phase space average over initial conditions to only one trajectory, with little loss of accuracy. It cannot get any better than this!

Finally, a trick that applies specifically to evaluating time correlation functions such as Eq. 1 is to (partially) combine the two time evolution operators$\exp (-i \hat{H} t / \hbar)$, which propagates from 0 to $t$, and $\exp (+i \hat{H} t / \hbar)$, which propagates from $t$ back to 0 -into one "forwardbackward" (FB) SC-IVR propagator (55-58); this is possible because operator $\hat{B}$ in Eq. 1 typically involves only a few degrees. The FB trick is carried out by performing a stationary phase approximation at time $t$, which effectively ties the forward and backward trajectories together, making one forwardbackward trajectory. The practical importance of this FB trick is that most of the oscillatory structure in the integrand is cancelled out analytically - because many degrees of freedom in a large system approximately back-integrate and make little net contribution to the phase of the integrand-rather than having to achieve this cancellation numerically.

It is perhaps most useful here simply to show an example of how the FB-IVR works and what it is capable to doing. The example is a simple model (59) of a timely topic, namely real-time molecular structure (e.g., femtosecond x-ray diffraction). The model system is a diatomic molecule (i.e., a Morse potential) in some condensed phase environment, e.g., a cluster, a solvent, a solid, a protein, etc., modeled by a set of harmonic oscillators (the ubiquitous "harmonic bath"). The Hamiltonian is thus of the form

$$
\begin{aligned}
H(p, r, \mathbf{P}, \mathbf{Q})= & \frac{p^{2}}{2 m}+V(r)+\sum_{k} \frac{1}{2} P_{k}^{2} \\
& +\frac{1}{2} \omega_{k}^{2}\left(Q_{k}+\frac{c_{k} r}{\omega_{k}^{2}}\right)^{2},
\end{aligned}
$$

where $\left\{\omega_{k}\right\}$ are the frequencies of the harmonic ("bath") modes. The initial state of the diatomic molecule, $|\phi\rangle$, is assumed to created by a Franck-Condon excitation (e.g., via a short laser pulse) from the ground state to an excited electronic state [for which $V(r)$ above is the potential energy function], and the harmonic modes describing the environment are initially in a Boltzmann distribution. The radial distribution function of the diatom (i.e., the molecular structure) at time $t$ later, $P_{t}(r)$, is given by the correlation function in Eq. 1 with operators $\hat{A}$ and $\hat{B}$ given by

$$
\begin{aligned}
& \hat{A}=|\phi\rangle\langle\phi| e^{-\beta \hat{H}_{b}} \\
& \hat{B}=\delta(r-\hat{r}),
\end{aligned}
$$

where $\hat{H}_{b}$ is the Hamiltonian for the harmonic modes alone. The FB-IVR procedure requires a Fourier expansion of operator $\hat{B}$, so that $P_{t}(r)$ is given by (1d) Fourier integral

$$
P_{t}(r)=(2 \pi \hbar)^{-1} \int_{-\infty}^{\infty} d p e^{-i p r / \hbar} \tilde{P}_{t}(p),
$$

[8a]

where the "structure factor" $\tilde{P}_{t}(p)$ is given by

$$
\tilde{P}_{t}(p)=\operatorname{tr}\left[\hat{A} e^{i \hat{H} t / \hbar} e^{i p \hat{r} / \hbar} e^{-i t \hat{H} / \hbar}\right] .
$$

Eqs. 8a and $\mathbf{8 b}$ entail no approximation, but now we use the FB-IVR to approximate $\tilde{P}_{t}(p)$,

$$
\begin{aligned}
\tilde{P}(p)= & (2 \pi \hbar)^{-F} \int d p_{0} \int d x_{0} \int d \mathbf{P}_{0} \\
& \cdot \int d Q_{0}\left\langle p_{0}, r_{0} \mid \phi\right\rangle\left\langle\phi \mid p_{0}^{\prime}, r_{0}^{\prime}\right\rangle \\
& \cdot\left\langle\mathbf{P}_{0}, \mathbf{Q}_{0}\left|e^{-\beta \hat{H}_{b}}\right| \mathbf{P}_{0}^{\prime}, \mathbf{Q}_{0}^{\prime}\right\rangle C_{0} \\
& \cdot\left(p_{0}, r_{0}, \mathbf{P}_{0}, \mathbf{Q}_{0}\right) e^{i S_{0}\left(p_{0}, r_{0}, \mathbf{P}_{0}, \mathbf{Q}_{0}\right) / \hbar} .
\end{aligned}
$$

Here, $\left(\mathrm{p}_{0}, \mathrm{r}_{0}, \mathbf{P}_{0}, \mathbf{Q}_{0}\right)$ are the initial conditions of all degrees of freedom for a classical trajectory that is evolved in the usual way from time 0 to $t$, at which point there is a momentum jump in the $r$ degree of freedom

$$
p_{t} \rightarrow p_{t}+p,
$$

with all other variables being continuous at $t$, and the trajectory is then propagated back to time 0 , with $\left(p_{0}^{\prime}, r_{0}^{\prime}, \mathbf{P}_{0}^{\prime}\right.$, $\mathbf{Q}_{0}^{\prime}$ ) being the final values. $S_{0}$ is the classical action for the forward-backward trajectory, and $C_{0}$ is the appropriate prefactor. The important practical feature of the FB-IVR, Eq. 9, is that it involves only a single phase space average over initial conditions (rather than a double phase space average if a separate SC-IVR were used for each of the two propagators in the correlation function) and thus involves not much more computational effort than a classical calculation (i.e., typically a few thousand trajectories are sufficient for convergence). Even more important is the fact that the FB integrand in Eq. 9 is much less oscillatory than would be that of the 

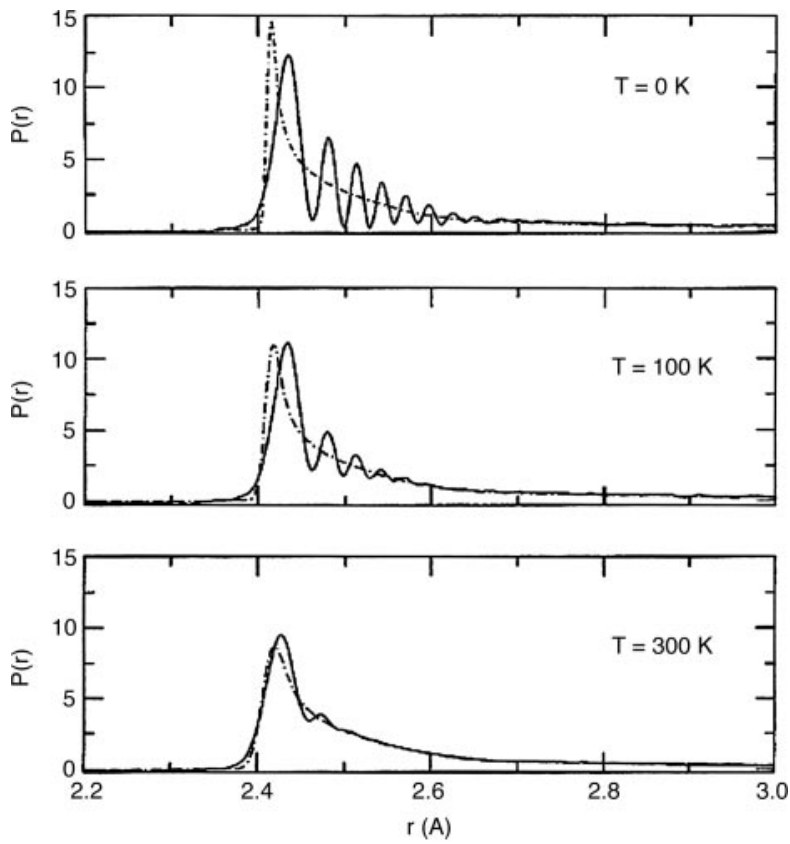

Fig. 1. The radial distribution function $P_{t}(r)$ of $I_{2}$ in a harmonic bath, at $t=192 \mathrm{fs}\left(\approx 1 \frac{1}{4}\right.$ vibrational periods after initial excitation); $T$ is the temperature of the harmonic bath. The solid line, which shows coherence structure, is the result of the forward-backward SC-IVR calculation, and the dash-dot curve is the result of a more approximate version (essentially classical mechanics with a Wigner distribution of initial conditions), which is clearly not able to describe these quantum features.

double phase space average because the momentum jump in Eq. 10 involves only the "solute" degree of freedom, so that bath degrees of freedom have their contribution to the FB action integral $S_{0}$ partially cancelled out.

Fig. 1 shows typical results (see ref. 59 for more details and more results): $P_{t}(r)$ is shown for $t=192 \mathrm{fs}$ (about 1/4 vibrational periods of $I_{2}$, the parameters used for the model). When the temperature of the bath is $T=0$-i.e., the bath is "frozen out" and has essentially no effect-one sees very pronounced interference (or coherence) structure, arising of course because the initial state of the diatomic molecule (compressed on the

1. Makri, N. (1995) J. Math Phys. 36, 2430-2457.

2. Nightingale, M. P. \& Umrigar, C. J., eds. (1999) Quantum Monte Carlo Methods in Physics and Chemistry (Kluwer, Dordrecht, The Netherlands).

3. Allen, M. P. \& Tildesley, D. J. (1987) Computer Simulation of Liquids (Clarendon, Oxford).

4. Frenkel, D. \& Smit, B. (1996) Understanding Molecular Simulation: From Algorithms to Applications (Academic, San Diego).

5. Kim, J. G., Fukumishi, Y., Kidern, A. \& Nakamura, H. (2004) J. Chem. Phys. 121, 5590-5601.

6. Berne, B. J. \& Harp, C. D. (1970) Adv. Chem. Phys. 17, 63-227.

7. Thirumalai, D. \& Berne, B. J. (1984) J. Chem Phys. 81, 2512-2513.

8. Miller, W. H., Schwartz, S. D. \& Tromp, J. W. (1983) J. Chem. Phys. 79, 4889-4898.

9. Thirumalai, D. \& Berne, B. J. (1983) J. Chem Phys. 79, 5029-5033.

10. Yamashita, K. \& Miller, W. H. (1985) J. Chem Phys. 82, 5475-5484. repulsive wall) is a coherent superposition of many vibrational eigenstates that each evolve coherently and, due to anharmonicity, get out of phase. The dash-dot line in Fig. 1 shows the result of a more approximate treatment (the linearized SC-IVR), which includes some quantum effects (in the distribution of initial conditions) but is clearly not capable of describing these coherence effects in the dynamics. As the temperature is increased (proceeding from Top to Bottom in Fig. 1), one sees that the coherence structure is progressively quenched, and by the time it is almost completely quenched (Bottom, $T=300$ $\mathrm{K})$, the classical-like approximation has

11. Rabani, E., Krilov, G. \& Berne, B. J. (2000) J. Chem. Phys. 112, 2605-2614.

12. Miller, W. H., Zhao, Y., Ceotto, M. \& Yang, S. (2003) J. Chem. Phys. 119, 1329-1342.

13. Zhao, Y., Yamamoto, T. \& Miller, W. H. (2004) J. Chem. Phys. 120, 3100-3107.

14. Yamamoto, T. \& Miller, W. H. (2005) J. Chem. Phys. 122, 044106.1-044106.13.

15. Ben-Nun, M., Quenneville, J. \& Martinez, T. J. (2000) J. Phys. Chem. A 104, 51615175 .

16. Kim, S. Y. \& Hammes-Schiffer, S. (2003) J. Chem. Phys. 119, 4389-4398.

17. Wang, H. \& Thoss, M. (2003) J. Phys. Chem. A 107, 2126-2136.

18. Nguyen, P. H. \& Stock, G. (2003) J. Chem. Phys. 119, 11350-11358.

19. Wu, Y. \& Batista, V. S. (2004) J. Chem. Phys. 121, 1676-1680.

20. Shalashilin, D. V., Child, M. S. \& Clary, D. C. (2004) J. Chem. Phys. 120, 5608-5615. become quite good. (One can equally well do the calculations as a function of the coupling strength between the diatomic and the bath, for a fixed temperature, and similar behavior is observed as the coupling strength is increased.)

The results seen in this example are precisely what one would expect: Quantum coherence is progressively quenched as the temperature of the bath (or the coupling to it) is increased, and if it is fully quenched, then classical mechanics becomes a good approximation. The point of the exercise is that one is able to simulate this behavior quite reliably via this FB-SC-IVR methodology in a model system with many degrees of freedom. There is nothing that would prevent a similar calculation (or that of any correlation function) for a more realistic model of a "real" molecular system, other than the increased computer time necessary for computing trajectories with a more realistic (and therefore complicated) potential energy surface. One would then be in the situation of having the simulation tell one whether quantum effects matter a lot, a little, or not at all, rather than simply having to assume that they do not matter (as one is doing when using only classical MD simulation).

This example (and others like it) provides "proof of principle" for the practicality of using semiclassical theory to add quantum effects to classical MD simulations of complex molecular systems. There is of course much work to be done to make this a full reality, but the way is reasonably clear.

This work was supported by the Director, Office of Science, Office of Basic Energy Sciences, Chemical Sciences, Geosciences, and Biosciences Division, U.S. Department of Energy (under Contract DE-AC0376SF00098) and by National Science Foundation Grant CHE-0345280.

21. Meyer, H. D. \& Worth, G. A. (2003) Theor. Chem Acc. 109, 251-267.

22. Shi, Q. \& Geva, E. (2004) J. Chem. Phys. 121, 3393-3404.

23. Wright, N. J. \& Makri, N. (2003) J. Chem. Phys. 119, 1634-1642.

24. Donoso, A. \& Martens, C. C. (2002) J. Chem. Phys. 116, 10598-10605.

25. Prezhdo, O. V. \& Pereverzev, Y. V. (2000) J. Chem. Phys. 113, 6557-6565.

26. Hone, T. D. \& Voth, G. A. (2004) J. Chem. Phys. 121, 6412-6422.

27. Bonella, S. \& Coker, D. F. (2001) J. Chem. Phys. 114, 7778-7789.

28. Cardenas, A. E., Krems, R. \& Coalson, R. D. (1999) J. Phys. Chem. A 103, 9469-9474.

29. Turi, L. \& Rossky, P. J. (2004) J. Chem. Phys. 120, 3688-3698.

30. Nest, M. \& Saalfrank, P. (2001) Chem. Phys. 268, 65-78.

31. Jan, S. \& Cao, J. (2001) J. Chem. Phys. 114, 9959-9968. 
32. Sergi, A., Kernan, D. M., Ciccotti, G. \& Kapral, R. (2003) Theor. Chem. Acc. 110, 49-58.

33. Makarov, D. E. \& Metiu, H. (1999) J. Chem. Phys. 111, 10126-10136.

34. Zhang, J. \& Pollak, E. (2003) J. Chem. Phys. 119, 11058-11063.

35. Antoniou, D. \& Schwartz, S. D. (1999) J. Chem Phys. 110, 7359-7364.

36. Gao, J. \& Truhlar, D. G. (2002) Annu. Rev. Phys. Chem. 53, 467-505.

37. Olsson, M. H. M., Siegbahn, P. E. M. \& Warshel, A. (2004) J. Am. Chem. Soc. 126, 2820-2828.

38. Craig, I. R. \& Manolopoulos, D. E. (2004) J. Chem. Phys. 121, 3368-3373.

39. Miller, W. H. (1974) Adv. Chem. Phys. 25, 69-177.

40. Miller, W. H. (1975) Adv. Chem. Phys. 30, 77-136.
41. Miller, W. H. (1970) J. Chem. Phys. 53, 35783587.

42. Heller, E. J. (1991) J. Chem. Phys. 94, 2723-2729.

43. Heller, E. J. (1991) 95, 9431-9432.

44. Miller, W. H. (1991) J. Chem. Phys. 95, 9428-9430.

45. Miller, W. H. (2001) J. Phys. Chem. A 105, 2942 2955.

46. Herman, M. F. \& Kluk, E. (1984) Chem. Phys. 91, 27-34.

47. Kay, K. G. (1994) J. Chem. Phys. 100, $4377-$ 4392.

48. Kay, K. G. (1994) J. Chem. Phys. 100, 4432-4445.

49. Doll, J. D., Freeman, D. L. \& Beck, T. L. (1990) Adv. Chem. Phys. 78, 61-126.

50. Makri, N. \& Miller, W. H. (1987) Chem. Phys. Lett. 139, 10-14.

51. Filinov, V. S. (1986) Nucl. Phys. B271, 717-725.
52. Wang, H., Manolopoulos, D. E. \& Miller, W. H. (2001) J. Chem. Phys. 115, 6317-6326.

53. Kaledin, A. L. \& Miller, W. H. (2003) J. Chem. Phys. 118, 7174-7182.

54. Kaledin, A. L. \& Miller, W. H. (2003) 119, 3078 3084.

55. Makri, N. \& Thompson, K. (1998) Chem. Phys. Lett. 291, 101-109.

56. Makri, N. \& Thompson, K. (1999) J. Chem. Phys. 110, 1343-1353.

57. Miller, W. H. (1998) Faraday Disc. Chem. Soc. 110, $1-21$

58. Sun, X. \& Miller, W. H. (1999) J. Chem. Phys. 110 6635-6644.

59. Wang, H., Thoss, M., Sorge, K., Gelabert, R., Gimenez, X. \& Miller, W. H. (2001) J. Chem. Phys. 114, 2562-2571. 